\section{When Less Is More}

\author{
The Editors, BioTechniques
}

bioeditor@biotechniques.com
$\mathrm{O}$ ne of the greatest challenges when writing a primary research article for a multidisciplinary scientific journal is making the information and discussion as accessible and interesting as possible to readers from a variety of research specialties. But this goal also has to be offset by the need to highlight novel conclusions and explain their broader implications within a specific field. At BioTechniques, the editors understand how challenging this can be for researchers, and we strive to help our authors present their cutting-edge methods and techniques precisely and succinctly to the journal's broad-based methods-oriented readership. In an attempt to further enhance these efforts, we have decided to alter our research article format beginning with our January 2010 issue.

Historically, BioTechniques has published research papers in three different categories: "Benchmarks," "Short Technical Reports," and "Research Reports." The original concept for the Benchmarks category revolved around the idea of presenting novel methods or substantive modifications to existing methods that could be explained in under 1000 words using only one or two figures and/or tables, while Short Technical Reports and Research Reports were intended to be more complex descriptions of new methods or techniques requiring up to 2500 or 4000 words, respectively, with anywhere from three to seven figures and/or tables. Although having these three article types does provide our authors a choice of venues in which to publish their research, the editors have concluded over the years that the difference between the methods reported in Short Technical Reports versus those in Research Reports is minor in many cases. This often creates a problem for authors and editors when it comes to deciding which category is best for a particular manuscript. In keeping with our goal to deliver the most pertinent information possible in a concise, engaging format, we have therefore decided to merge the Short Technical Reports and Research Reports into a single new category that will simply be called "Reports."
Reports will have a 3000-word limit within the main text of the article, along with three to four supporting figures and/or tables. Any additional content required to support the findings presented in a Report-including additional protocols, figures, tables, and illustrations - may be placed in supplementary material files, which will accompany the article on our web site. With regard to article structure, Reports will follow the Short Technical Report format with a combined Results and Discussion section to enable authors to directly relate the full implications of their findings to readers in one conclusive section. Benchmarks will continue to relate novel methods or significant modifications to existing methods using their current format, while Reports will now serve to describe more in-depth and detailed techniques and methodologies.

We feel strongly that publishing two categories of research articles will enhance not only the BioTechniques reading experience, but also aid our authors as they prepare their manuscripts for submissions - providing a format that will enable focused, analytical results and conclusions bolstered by high-quality figures, illustrations, and a variety of supplementary online content.

A new, abbreviated guide for authors can be found in this issue of BioTechniques, while a more comprehensive version-detailing specific length and style requirements, figure specifications, reference format, and necessary forms and statements, as well as a general description of the review process-can be found on our web site, www.biotechniques.com. In the end, we are confident that the new Report format will have a positive impact for both our authors and readers going forward. As always, we welcome all your comments and feedback through email to the editors at bioeditor@ biotechniques.com, or online by posting to our Molecular Biology Forums under "To the Editor" (http://molecularbiology.forums. biotechniques.com). TGh

BioTechniques 47:995 (December 2009)

doi 10.2144/000113309
"At BioTechniques, the editors strive to help our authors present their cutting-edge methods and techniques precisely and succinctly to the journal's broad methodsoriented readership." 